Bangladesh J. Sci. Res. 27(1): 27-38, 2014 (June)

\title{
COMPETING RISKS ANALYSIS OF UNDER-FIVE CHILD MORTALITY IN BANGLADESH
}

\author{
Tasnuva Tabassum ${ }^{*}$ and Wasimul Bari ${ }^{1}$ \\ Department of Disaster Science and Management, University of Dhaka, \\ Dhaka-1000, Bangladesh
}

\begin{abstract}
This study implements an analysis of under five mortality to the Bangladesh Demographic and Health Survey, 2011 data from a competing risks perspective. Kaplan - Meier overall survival curves along with log-rank test p-values are employed to determine the prospective covariates for the Cox proportional hazard (PH) model. Later the typical Cox PH model is used to model the causespecific hazard for the two competing causes, namely disease and non-disease on the selected covariates. It is revealed that mother's primary and secondary education, birth order of the index child in case of disease model; mother's higher education, wealth index of the index child in case of non-disease model and mothers aged greater than 30 years at the time of giving birth, home as a place of child delivery in both model played significant role in decreasing under five child mortality.
\end{abstract}

Key words: Competing risks, under-five mortality, Cox PH model, KaplanMeier survival probabilities, log-rank test

\section{Introduction}

Reducing child mortality rate is referred as the fourth of the eight millennium development goals (MDGs), for which Bangladesh needs to reduce the mortality to approximately less than 50 per 1000 live births by 2015 (ICDDR,B 2007). The eight millennium goals, ranging from eradicating extreme poverty and hunger, achieving universal primary education, promoting gender equality and empowering women, reducing child mortality, improving maternal health, halting the spread of HIV/AIDS, malaria and other diseases, ensuring environmental sustainability to developing a global partnership for development by the year 2015; are the international goals agreed to achieve by the 189 member countries of the United Nations and 23 world's leading development institutions at the millennium summit in 2000. Among these, the fourth goal (MDG-4) can be interpreted in a more elaborative way as: reducing the under-five mortality by two-thirds between 1990 and 2015. In Bangladesh, the under-five mortality rate at the beginning of the MDG period was 133 per 1000 live births (ICDDR,B 2007),

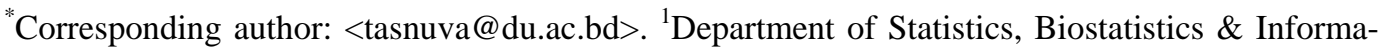
tics, University of Dhaka, Dhaka-1000, Bangladesh.
} 
which currently is 53 per 1000 live births (BDHS 2011). This study takes "under-five” mortality in Bangladesh as an event which may occur due to either disease (child died either from neonatal tetanus, measles, diarrhea, meningitis or neonatal jaundice) or nondisease causes (child died either by congenital abnormalities, birth asphyxia, drowning, birth injury, respiratory distress, premature birth, serious infection, malnutrition or other causes). The identification of the risk factors attributable to the "under-five" mortality is of special importance to meet the challenge of reducing child mortality through setting priorities. Since competing risk setup is a complicated situation having more than one cause of failure, special care should be taken care of, as applying usual survival techniques in the presence of competing risks without modification is not worthy.

Adepoju et al. (2012) found that mother's secondary and higher education, mother's age at first birth, place of delivery, child ever breastfed, type of birth, gender of child influences child mortality the most in rural Nigeria. The integrated management of childhood illness (IMCI), launched by WHO and UNICEF in the mid-1990s, could not affect the overall yearly rate of child mortality in IMCI and comparison areas although the last two years of the study showed $13.4 \%$ lower mortality rate in IMCI than that in comparison areas (Arifeen et al. 2009). Chowdhury (2013) showed that father's education, place of residence, region of residence, number of children under five years of age, previous death of sibling, mother's age and breastfeeding can significantly influence "under-five" mortality. Islam et al. (2010), on the other hand, found that parents education and occupation, type of latrine and electricity influence infant and child mortality while only mother's education and occupation influence post-neonatal mortality. ICDDR,B (2007), as a part of its zinc supplementation program, showed that zinc treatment for diarrhea, along with exclusive breastfeeding, proper complimentary feeding, vitamin A program and current vaccine is crucial for reducing "under-five" mortality. Siddiqi et al. (2011) showed that parent's education, birth order, birth interval, wealth index, mother's age at marriage, access to improved toilet facilities and source of drinking water play important role in influencing child mortality. Longer preceding birth interval, primary healthcare, childhood immunization and access to tube-well water were found by Mozumder et al. (1998) as the significant factors for improving child survival. Mondal et al. (2009) found that immunization, ever breastfeeding, mother's age at birth and birth interval are the most important determinants of neonatal, post-neonatal and child mortality. Whereas Rahman and Sarkar (2009) found that area of residence, division, mother's education and antenatal care are responsible for child mortality. Lastly, father's education and occupation, breastfeeding status, birth order, timing of first antenatal checkup and tetanus toxoid (TT) during pregnancy were seen to influence the child mortality by Uddin et al. (2009) 
Many studies have been conducted for the determination of the risk factors of child as well as "under-five" mortality in Bangladesh, but none of those investigated the risk factors from a competing risks perspective. Therefore, this study aims at conducting a competing risks analysis of the child mortality data. To fulfill this purpose, the influencing risk factors are investigated in this study implementing the Cox proportional Hazard (PH) model for child mortality in case of both disease and non-disease causes.

\section{Methods}

For the purpose of this paper, data were analyzed in two steps. First, Kaplan-Meier survival curves were employed for doing bivariate analysis. The variables found to be statistically significant in the bivariate analysis were then put to competing Cox proportional hazard model. This section briefly describes Kaplan-Meier survival curve and competing Cox PH model.

Kaplan-Meier survival curves: For this study, the event of interest was the death of children aged under five years due to either disease or non-disease causes. Assume that the events occur at the ordered time points $t_{1}\left\langle t_{2}\left\langle\Lambda\left\langle t_{k}\right.\right.\right.$. Now if $d_{j}$ denote the number of events occurred at time $t_{j} ; j=1,2, \Lambda k$ and $n_{j}$ denotes the number of individuals at risk at that time; then the Kaplan-Meier estimate of the survival probability at time $t_{j}$ is as follows:

$$
\hat{S}\left(t_{j}\right)=\prod_{i=1}^{j}\left(1-\frac{d_{j}}{n_{j}}\right) .
$$

The Kaplan-Meier survival probabilities are estimated for each of the categories of a covariate and then plotted in the same graph. The log-rank test p-value is provided along with the survival curve. For this study, the survival curves for mother's age at birth, place of residence, mother's education, wealth index, place of delivery, birth order: first child or not and gender of child are provided along with discussion.

Competing Cox proportional hazard (PH) model: The reason for implementing Cox PH model is it's flexibility and robustness. In competing risk setup, it models the effects of covariates on the cause-specific hazard function. According to Cox (1972), for the $j^{\text {th }}$ cause the model stands as:

$$
h_{j}\left(t_{i} \mid x_{i}\right)=h_{0 j}\left(t_{i}\right) e^{\beta_{j}^{\prime} x_{i}},
$$


$h_{j}\left(t_{i} \mid x_{i}\right)$ being the hazard at time $t_{i}$ due to cause $j, h_{0 j}\left(t_{i}\right)$ being the baseline hazard at time $t_{i}$ due to cause $j, \beta_{j}$ being the regression coefficient vector due to cause $j$ and $x_{i}$ being the $p$ vector covariate.

The overall likelihood function of Cox proportional hazard model under competing risk setup is as follows:

$$
L_{j}(\beta)=\prod_{i=1}^{k} \frac{e^{\beta_{j}^{\prime} X_{l(i j)}}}{\sum_{l \in R_{i j}} e^{\beta_{j}^{\prime} X_{l}}}
$$

where $L_{j}(\beta)$ is the likelihood function for the $j^{\text {th }}$ cause and $\frac{e^{\beta_{j}^{\prime} X_{l(i j)}}}{\sum_{l \in R_{i j}} e^{\beta_{j}^{\prime} X_{l}}}$ is the conditional probability that a particular individual $l(i j)$ would fail at time $t_{i j}$ due to cause $j$ given the risk set $R_{i j}$ and the fact that exactly one individual fails at time $t_{i j}$. It was assumed that the events due to $j^{\text {th }}$ cause occurred at observed distinct ordered time points $t_{1 j}\left\langle t_{2 j}\left\langle\Lambda\left\langle t_{k j}\right.\right.\right.$. The score function $U\left(\beta_{j}\right)$ stands as $U\left(\beta_{j}\right)=\frac{\delta}{\delta \beta_{j}^{\prime}} \ln L_{j}(\beta)$ and the estimating equation is $U\left(\hat{\beta}_{j}\right)=0$.

This estimating equation can be solved by Newton-Raphson iterative approach to obtain estimate for $\beta_{j}$. The estimate at the $v^{\text {th }}$ Newton-Raphson iteration step is as follows:

$$
\hat{\beta}_{j}^{(v)}=\hat{\beta}_{j}^{(v-1)}+\left[I^{*}\left(\beta_{j}\right)\right]_{\beta_{j}=\hat{\beta}_{j}^{(v-1)}}^{-1}\left[U\left(\beta_{j}\right)\right]_{\beta_{j}=\hat{\beta}_{j}^{(v-1)}} ; v=1,2,3 \Lambda .
$$

Here, $I^{*}\left(\beta_{j}\right)=-\frac{\delta}{\delta \beta_{j}^{\prime}} U\left(\beta_{j}\right)$ is the observed information matrix.

\section{Results}

Data have been taken from the 2011 BDHS (Bangladesh Demographic Health Survey) for the current study. Children aged less than five years are considered only where the age is constructed by taking the difference between the date of interview and the date of birth of the index child. Since the last five births of every woman having at least one child have been considered, children having same mother provide the same information regarding their mother. For the purpose of the current study, only two causes are considered to be responsible for under-five mortality which are: disease and non-disease. 
In total 8753 children have been considered of which 410 (4.68\%) died. Among these 410 dead children, 127 died from disease and the rest 283 died by non-disease causes. The alive children are considered as censored observations. Mother's education level (no education, primary, secondary, higher), mother's age at birth of the index child $(<20,20$ $30,>30$ ), wealth index of the household (poor, middle-class, rich), place of residence (urban, rural), sex of the index child (male, female), place of delivery (home, hospital and other) and birth order of the index child (first birth, other) are some of the explanatory variables that are taken into account.

In the data, 1687 (19.27\%) children were found to have mothers who did not have any education at the time of their births. Whereas 2684 (30.66\%), 3690 (42.16\%) and 692 (7.91\%) children were found to have mothers who had had primary, secondary and higher education at the time of their births, respectively. Among 8753 children 3646 (41.65\%) came from poor families, 1661 (18.98\%) from middle-class families and 3446 (39.37\%) from rich families. And among the total sample, 2674 (30.55\%) were taken from urban areas and 6079 (69.45\%) from rural areas. In 1777 (20.30\%) cases, children were found to have mothers who had been less than 20 years of age at the time of their children's births. Whereas in 5280 (60.32\%) and 1696 (19.38\%) cases children were found to have mothers who had been 20-30 and greater than 30 years of age at the time of their children's births, respectively. 4502 (51.43\%) male and 4251 (48.57\%) female children were considered in the data. 6351 (72.56\%) children were delivered in home and 2402 (27.44\%) in hospital and other places. Finally, 3122 (35.67\%) children were found to be their parents' first child and 5631 (64.33\%) of them were found to be their parents' second or other child.

Bivariate analysis: From the survival curves (Fig. I), it is apparent that children having mothers whose age was greater than 30 at the time of their births have the highest survival. Children having mothers whose age was between 20 and 30 at the time of their births show lower survival than their previous counterparts. And finally, the children having mothers whose age was below 20 at the time of their birth have the lowest survival probabilities. The log rank test shows a p-value of zero for this variable, which means that the three categories of the variable "mother's age at birth" are significantly different from each other. Children having mothers with no, primary, secondary and higher education have different survival probabilities shown in different survival curves, those vary significantly (Log-rank test p-value: 0.000) from one another. In contrast, children from urban and rural areas showed no difference (Log rank test p-value: 0.242) in their survival probabilities. Parents' first children showed significant higher survival probabilities (Log rank test p-value: 0.018) than those of others. Similarly, children delivered at home showed significant higher survival probabilities (Log rank test p-value: 0 ) than those delivered in hospitals or other places. Male and female children showed 

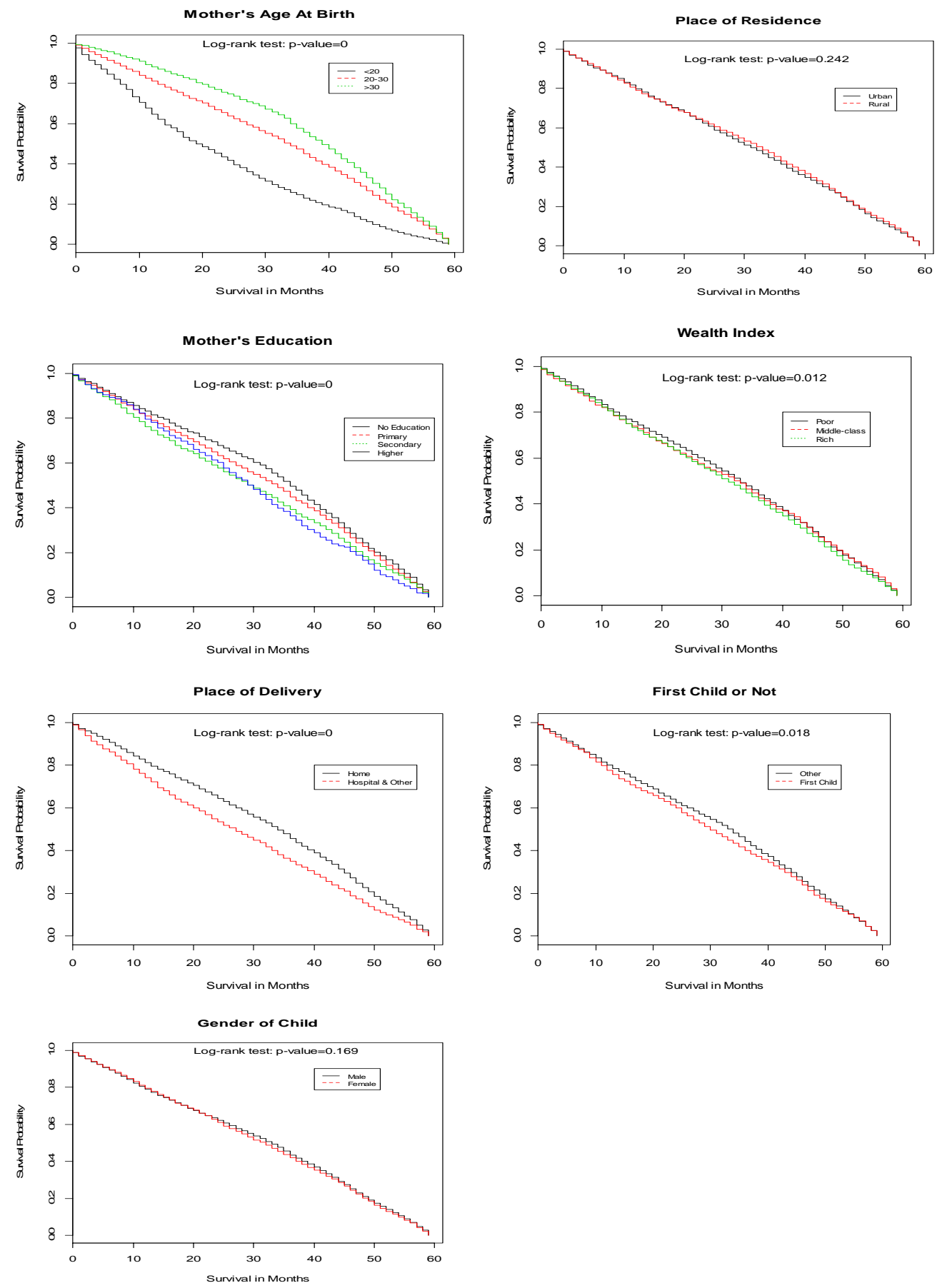

Fig. 1. Survival curves by each of the covariates along with their log-rank test p-values. 
no difference in their survival probabilities (Log rank test p-value: 0.169). Children from poor, middle-class and rich families; on the other hand, have different survival probabilities (Log rank test p-value: 0.012). The variables "mother's age at birth", "mother's education”, "wealth index", "place of delivery" and "birth order: first child or not" have very low log rank test p-value which means that the children's survival probabilities vary significantly among the categories of these variables. But since the variables "place of residence" and "gender of child" show insignificant results, they are discarded from the Cox proportional hazard model.

Competing Cox proportional hazard model: The parameter estimates obtained from the competing Cox proportional hazard model due to disease and that due to non-disease are presented in Table 1. The estimates indicate that there exists strong evidence that children having mothers whose age was less than 20 at the time of their births had a higher rate of mortality due to disease than those having mothers whose age was between 20 and 30 at the time of their births. The hazard ratio also indicate that mothers aged below 20 years tend to give births to children who have 2.568 times risk of mortality due to disease than their counterparts having older mothers. But the children having mothers whose age was greater than 30 at the time of their birth have lower rate of mortality due to disease than those having mothers whose ages were between 20 and 30 at the time of their births. A reason for this may be mother's maturity. Maturity increases with age. Therefore, older mothers can take greater care of children which may result in their higher survival probabilities. Another reason may be that fertility decreases with the increase of age. Therefore, aged mothers tend to have fewer children and can spend much time behind each child for proper care. The competing Cox proportional hazard model due to non-disease also shows that mothers aged below 20 years during giving birth tend to have children whose mortality rate is significantly higher whereas mothers aged above 30 years during giving birth tend to have children whose mortality rate is significantly lower; compared to the children having mothers aged 20-30 years at the time of giving birth. The hazard ratios also indicate that children having mothers aged below 20 years at the time of giving birth have 2.220 times risk of mortality whereas children having mothers aged above 30 years at the time of giving birth have $32.4 \%$ less risk of mortality due to non-disease, compared to the children having mothers aged 20-30 years at the time of giving birth.

Mother's education plays an important role in reducing child mortality. From both competing Cox proportional hazard model due to disease and non-disease, it is apparent that women with higher education give births to children who have lower mortality rate than women with none (Table 1). 
Table 1. Parameter estimates from competing Cox proportional hazard model for child mortality due to disease and non-disease causes.

\begin{tabular}{|c|c|c|c|c|c|c|}
\hline \multirow[b]{2}{*}{ Variable } & \multicolumn{3}{|c|}{ Disease model } & \multicolumn{3}{|c|}{ Non-disease model } \\
\hline & $\hat{\beta}$ & HR & p-value & $\hat{\beta}$ & HR & $\mathrm{p}$-value \\
\hline \multicolumn{7}{|l|}{ Mother's age } \\
\hline$<20$ & 0.943 & 2.568 & 0.001 & 0.798 & 2.220 & 0.000 \\
\hline $20-30 *$ & - & - & - & - & - & - \\
\hline$>30$ & ÑD.109 & 0.897 & 0.628 & ÑD.391 & 0.676 & 0.032 \\
\hline \multicolumn{7}{|l|}{ Mother's education } \\
\hline No* & - & - & - & - & - & - \\
\hline Primary & $\tilde{\mathrm{N}} \mathrm{D} .420$ & 0.657 & 0.615 & $\tilde{N}$.041 & 0.960 & 0.812 \\
\hline Secondary & $\tilde{N} 0.852$ & 0.427 & 0.001 & $\tilde{N} 0.127$ & 0.880 & 0.483 \\
\hline Higher & ÑD.784 & 0.457 & 0.109 & ÑD.970 & 0.379 & 0.012 \\
\hline \multicolumn{7}{|l|}{ Economic status } \\
\hline Poor & ÑD.195 & 0.823 & 0.408 & 0.344 & 1.410 & 0.049 \\
\hline Middle-class* & - & - & - & - & - & - \\
\hline Rich & $\tilde{N}$. 410 & 0.663 & 0.112 & 0.088 & 1.092 & 0.625 \\
\hline \multicolumn{7}{|l|}{ Birth order } \\
\hline First birth & $\tilde{N}$ D.429 & 0.651 & 0.087 & 0.033 & 1.034 & 0.823 \\
\hline Other* & - & - & - & - & - & - \\
\hline \multicolumn{7}{|l|}{ Place of delivery } \\
\hline Home & $\tilde{N} 0.484$ & 0.616 & 0.040 & ÑD.632 & 0.531 & 0.000 \\
\hline Hospital \& other* & - & - & - & - & - & - \\
\hline
\end{tabular}

*: Reference group

HR: Hazard ratio

Women with higher education are likely to have a better knowledge about child care. Therefore children with educated mothers enjoy a longer as well as healthier life.

Another reason may be that educated mothers participate more in decision making regarding childbirth. They tend to have fewer children than their uneducated counterparts. Therefore, they can spend adequate time for proper care of each child. However, the competing Cox proportional hazard model due to disease shows significant results for only children having mothers with primary and secondary education. The risk of mortality due to disease of children having mothers with primary and secondary education are $34.3 \%$ and $57.3 \%$ less than that of children having mothers with no education, respectively. The competing Cox proportional hazard model due to nondisease, on the other hand, shows significant result for only children having mothers with 
higher education. The result indicates that children having mothers with higher education have $62.1 \%$ less risk of mortality due to non-disease than the children having mothers with no education.

Birth order of the index child also plays an important role in influencing child mortality. The competing Cox model due to disease shows that if the baby is the first child of his/her parents, she/he has a lower mortality rate due to disease than the child who is second one or later in birth line. The reason may be that the parents pay an extra attention to the mother's nutrition when they expect their first child as well as to the child's nutrition after the birth of the child. Mothers go to the doctors for regular prenatal and postnatal checkup. This results in lower risk (in this study, 34.9\% less risk) of mortality due to disease among first child than among second or later child in birth line. Surprisingly, the competing Cox model due to non-disease shows the opposite result. It says that the first child has a higher mortality rate due to non-disease than the second or later child at birth line. People lack experience in handling children carefully when they become parents for the first time. This lack of experience leads to the child death regardless of the matter that extra care was taken on maternal and child nutrition. In this study, the first child has 1.034 times risk of mortality due to non-disease than the second or later child in birth line.

The wealth index of the household of index child shows no significant impact on the child mortality due to disease. But the competing Cox model due to non-disease shows that poor people have higher rate of child mortality due to non-disease than the middle class people. Middle class people can afford the maintenance fee for the proper care of the children which is not possible for poor people. Therefore children of poor people tend to die by non-disease causes in higher proportion than those of middle class families. In this study, poor children have $41 \%$ higher risk of mortality due to non-disease than middle class children.

Generally, it is considered that children delivered in hospitals live a longer life than those delivered in home since hospitals are more hygienic and secured place for delivery. But this study shows an interesting result. Both the competing Cox model due to disease and non-disease reveals that the children born in home tend to survive for a longer time than the children born in hospitals, clinics or places other than home. This result embraces a special situation of Bangladesh. People in Bangladesh do not go to the hospital for any kind of regular checkup until it becomes a serious situation. Most of the pregnant women, specially in rural areas, do not go to the hospital for their regular checkup or child delivery until severe illness occur. Therefore, pregnant women arriving at hospitals for delivery are found to be very sick or at the last stages of their lives when doctors have very little time to save these women's as well as their children's lives. This 
leads to the child mortality rate higher in hospitals than in home. In this study, children delivered at home are found to have $38.4 \%$ less risk of mortality due to disease and $46.9 \%$ less risk of mortality due to non-disease, compared to the children delivered at hospital, clinics or places other than home.

\section{Conclusion}

From bivariate analysis, the explanatory variables- mother's education, mother's age at birth, wealth index, place of delivery and birth order of the index child are found to provide significant results. Therefore, only they are employed in the competing Cox PH model. Among these variables, mother's age at birth, mother's education and place of delivery show strong effects on child mortality rate in both disease and non-disease model. The discrepancy between the two models occurs in case of wealth index and birth order of the index child. First child of parents enjoy longer life since their mortality rate is lower due to disease than the child who is later in birth line. But for non-disease model, the mortality rate of first child becomes higher than other children. The mortality rate of poor children because of non-disease causes is higher than their richer counterparts. Clearly the results indicate that for reducing child mortality or in other sense, improving child survival; mother's education level should be increased, poverty level must be reduced, awareness should be created regarding child marriage and mother's regular prenatal and postnatal checkup in hospital, clinics or health complex. Therefore, policy makers should give importance and set their priorities on these issues in order to achieve the goal of meeting the challenge of MDG-4.

\section{References}

Adepoju, A.O., O. Akkani and A.O. Falusi. 2012. Determinants of child mortality in rural Nigeria. Cancer Biol. 2(2):7-14.

Arifeen, S.E., D. M. E. Hoque, T. Akter, M. Rahman, M.E. Hoque, K. Begum, E.K. Chowdhury, R. Khan, L. S. Blum, S. Ahmed, M. A. Hossain, A. Siddik, N. Begum, Q. S. Rahman, T. M. Haque, Sk M. Billah, M. Islam, R. A. Rumi, E. Law, Z. A. M. Helal, A. H. Baqui, J. Schellenberg, T. Adam, L. H. Moulton, J - P Habicht, R. W. Scherpbier, C. G. Victora, J. Bryce and R. E. Black. 2009. Effect of the integrated management of childhood illness strategy on childhood mortality and nutrition in rural area in Bangladesh: A cluster randomized trial. Lancet 374:393-403.

Bangladesh Demographic and Health Survey. 2011. National Institute of Population Research and Training (NIPORT). Calverton, Maryland and Dhaka, NIPORT, Mitra and Associates and Macro International inc.

Chowdhury, A.H. 2013. Determinants of under-five mortality in Bangladesh. Open J. Stat. 3: 213219.

Cox, D.R. 1972. Regression models and life tables (with discussion). J. Roy. Statist. Soc. Ser. B 34: 187-220. 
ICDDR,B. 2007. Reduce child mortality-MDG 4, ICDDR, B Annual Report. 2007. 24-33.

Islam, R., Q.H. Chowdhury and K. Hossain. 2010. Socio-economic determinants of neonatal, postneonatal, infant and child mortality. Int. J. Sociol. Anthropol. 2(6):118-125.

Mondal, M.N.I., M.K. Hossain and M.K. Ali. 2009. Factors influencing infant and child mortality: A case study of Rajshahi district, Bangladesh. J. Hum. Ecol. 26(1):31-39.

Mozumder, A. B. M. K. A., B. Khuda and T. T. Kane. 1998. Determinants of infant and child mortality in rural Bangladesh. ICDDR,B Working Paper No. 115. International Center for Diarrhoeal Disease Research, Bangladesh.

Rahman, K.M.M. and P. Sarkar. 2009. Determinants of infant and child mortality in Bangladesh. Pak. J. Soc. Sci. 6(3):175-180.

Siddiqi, M. N. A., M. N. Haque and Dr. M. A. Goni. 2011. Differentials and determinants of under-five mortality in Bangladesh. Int. J. Curr. Res. 3(3):142-148.

Uddin, M.J., M.Z. Hossain and M.O. Ullah. 2009. Child mortality in a developing country: A statistical analysis. J. Appl. Quantitative Method 4(3):270-283.

(Manuscript received on 11 May, 2014; revised on 07 September, 20014) 